\title{
On the Regime of Ground Water During Filtration from Channels in The Soil Layer with the Underlying Pressure Horizon
}

\author{
Bereslavsky E. N.*, Mkrtchyan B. A. \\ Saint Petersburg state University of civil aviation, Saint Petersburg, Russia.
}

*Corresponding Author: Bereslavskii Eduard Naumovich, Saint Petersburg State University of Civil Aviation, Russia.

\begin{abstract}
For solving the mixed multiparameter boundary value problem of the theory of analytical functions, the Polfubarinova-Kochina method is used as are the methods of the conformal map of circular polygons developed for fields inherent to the problem underground hydromechanics.
\end{abstract}

Keywords: filtration, ground water, differentional equations of Fuces, conformal mapping.

\section{INTRODUCTION}

In the hydrodynamic formulation, the problem of liquid filtration from a channel filled with water through a soil layer with an underlying pressure horizon of relatively high permeability in the presence of evaporation from the free surface of groundwater is solved.

When considering flows from channels, it is usually assumed [1-5] that filtration occurs only through their bottom, which is usually taken as a horizontal segment. Taking into account the influence of the depth of water in the channels, that is, the study of movement not only through the bottom, but also through the slopes of the channels makes an additional angular special point in the physical area, which significantly complicates the solution of the problem. In this paper, the method developed earlier [6] is used to study the regime of ground water when filtering from such channels filled with water, in the presence of evaporation from a free surface.

In the framework of the theory of plane steady filtration of an incompressible fluid according to Darcy's law deals with the flow of rectangular channel of width $2 \mathrm{l}$ with water depth $\mathrm{H}$ in the soil capacity $\mathrm{T}$, underlain by the well permeable pressurized horizon is relatively high permeability, the pressure of which is equal to $\mathrm{H} 0\left(0<\mathrm{H}_{0}<\mathrm{T}\right)$, in the presence of a uniform intensity of evaporation $\varepsilon(0<\varepsilon<1)$ with a free surface.

To study the flow formulates a combined multi-parameter boundary problem of the theory of analytic functions which is solved using the method of P.Y. Polubarinova-Kochina [1-5], based on the application of the analytic theory of linear differential equations of Fuchs class [7] and the method of integrating such equations with four regular singularities [8-10], which is characteristic for problems of underground hydromechanics [11-15]. It should be noted that taking into account the specific features of the movements under consideration allows you to get solutions to problems in a closed form through elementary functions, which makes its use the most simple, convenient and effective.

On the basis of the model the algorithm of calculation of the size of the zone of saturation and the desired filtration flow rate in the case where filtering of the channels have to evaluate the joint impact on the pattern of such important factors as the filtration capacity of the reservoir and backwater from the deep artesian horizon of relatively high permeability, channel width and depth of the water in the evaporation from the free surface of groundwater.

On the basis of the obtained exact analytical dependences and by means of numerical calculations, the hydrodynamic analysis of the influence of all the specified physical parameters of the studied model is carried out. We compare the results of mathematical modeling with the same filtration characteristics for the flow pattern in the case when $\mathrm{H}=0$, that is, when filtering only through the bottom of the channels. 


\section{REFERENCES}

[1] Polubarinova-Kochina P. Y. Theory of ground water movement. Moscow: Gostekhizdat, 1952. 676p.; 2nd edition. Moscow: Nauka, 1977. 664p.

[2] Aravin V. I., Numerov S. N. Theory of motion of liquids and gases in a non-deformable porous medium. Moscow: Gostekhizdat, 1953. 616p.

[3] Development of research on filtration theory in the USSR (1917-1967). Moscow: Nauka, 1969. 545p.

[4] Mikhailov G. K., Nikolaevsky V. N. Movement of liquids and gases in porous media / / Mechanics in the USSR for 50 years. Moscow: Nauka, 1970.Vol. 2.Pp. 585-648.

[5] Kochina P. Y. Hydrodynamics and filtration theory. Selected works. Moscow: Nauka, 1991. 351p.

[6] Bereslavsky E. N. On the application of the equations of the Fuchs class to calculate seepage from channels and sprinklers // Journal of Applied mathematics and mechanics. 2013. Vol. 77.Release.5.Pp. 711-724.

[7] Golubev V. V. Lectures on the analytical theory of linear differential equations. Moscow.; Leningrad. Gostekhizdat, 1950. 436 P.

[8] Bereslavsky E. N. On integration in a closed form of a class of Fuchsian equations and its application. Differential equations. 1989. Vol. 25.No. 6.Pp.1048-1050.

[9] Bereslavsky E. N. On differential equations of the Fuchs class related to the conformal mapping of circular polygons in polar grids / / Differential equations. 1997. Vol. 33.No. 3.Pp. 296-301.

[10] Bereslavsky E. N. On accounting for infiltration or evaporation from a free surface by the method of circular polygons / / Applied mathematics and mechanics. 2010. Vol. 74.Release.2.Pp. 239-251.

[11] Bereslavsky E. N., Kochina P. Y. On some equations of the Fuchs class in hydro-aerodynamics / / Proceedings of the Russian Academy of Sciences. Fluid and gas mechanics. 1992.No. 5.Pp. 3-7.

[12] Bereslavsky E. N., Kochina P. Y. On differential equations of the Fuchs class occurring in some problems of fluid mechanics / / proceedings of the Russian Academy of Sciences. Fluid and gas mechanics. 1997.No. 5.Pp. 9-17.

[13] Bereslavsky E. N., Kochina P. Y., Kochina N. N. Analytical theory of linear differential equations of the Fuchs class and some problems of underground hydromechanics. Preprint No. 567.Edition.1.Moscow: Institute of problems of mechanics of the Russian Academy of Sciences, 1996. 122p.

[14] Bereslavsky E. N. Modeling of filtration flows from channels / / Reports of the Russian Academy of Sciences. 2010. Vol. 34.No. 4.Pp. 472-475.

[15] Bereslavsky E. N. Modeling of filtration flows from channels / / Applied mathematics and mechanics. 2011. Vol. 75.Release.4.Pp. 563-571.

Citation: Bereslavsky E. N \& Mkrtchyan B. A (2020). On the Regime of Ground Water During Filtration from Channels in The Soil Layer with the Underlying Pressure Horizon. International Journal of Scientific and Innovative Mathematical Research (IJSIMR), 8(1), pp. 12-13. http://dx.doi.org/ 10.20431/2347 3142.0801002

Copyright: () 2020 Authors, this is an open-access article distributed under the terms of the Creative Commons Attribution License, which permits unrestricted use, distribution, and reproduction in any medium, provided the original author and source are credited. 